INFLAMMATORY BOWEL DISEASE

\title{
Therapeutic effect of urocortin and adrenomedullin in a murine model of Crohn's disease
}

\author{
E Gonzalez-Rey, A Fernandez-Martin, A Chorny, M Delgado
}

See end of article for authors' affiliations

Correspondence to

Correspondence to:
Dr M Delgado, Instituto de Parasitologia y

Biomedicina, CSIC, Avd.

Conocimiento, PT Ciencias de la Salud, Granada

18100, Spain;

mdelgado@ipb.csic.es

Revised version received 23 November 2005 Accepted for publication 28 November 2005

Published online first

9 January 2006
Background and aim: Urocortin 1 (UCN) and adrenomedullin (AM) are two recently discovered neuropeptides that, due to their distribution and binding to receptors in immune cells, have emerged as potential endogenous anti-inflammatory factors. Crohn's disease is a chronic debilitating disease characterised by a Th1 driven severe inflammation of the gastrointestinal tract. This study investigated the therapeutic effect of $U C N$ and $A M$ in a murine model of colitis.

Methods and results: Treatment with UCN or AM ameliorated significantly the clinical and histopathological severity of the inflammatory colitis, abrogating body weight loss, diarrhoea, and inflammation, and increased the survival rate of colitic mice. The therapeutic effect was associated with downregulation of both inflammatory and Th1 driven autoimmune responses, including regulation of a wide spectrum of inflammatory mediators. In addition, partial involvement of interleukin 10 secreting regulatory cells in this therapeutic effect was demonstrated. Importantly, UCN or AM treatments were therapeutically effective in established colitis and avoided recurrence of the disease.

Conclusions: This work identifies UCN and AM as two potent anti-inflammatory factors with the capacity to deactivate the intestinal inflammatory response and restore mucosal immune tolerance at multiple levels. Consequently, both peptides represent novel multistep therapeutic approaches for the treatment of Crohn's disease and other Th1 mediated inflammatory diseases.

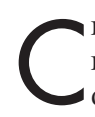
rohn's disease (CD) is a chronic, idiopathic, and relapsing inflammatory bowel disease characterised by dysfunction of mucosal $\mathrm{T}$ cells, altered cytokine production, and cellular inflammation that ultimately leads to damage of the distal small intestine and colonic mucosa, resulting in a range of gastrointestinal and extraintestinal symptoms-that is, abdominal pain, rectal bleeding, diarrhoea, weight loss, skin and eye disorders, and delayed growth and sexual maturation in children. ${ }^{12}$ Although its aetiology remains unknown, there is circumstantial evidence to link $\mathrm{CD}$ to failure of the mucosal immune system to attenuate the immune response to endogenous antigens. ${ }^{1}$ Several animal models of CD have been developed recently. ${ }^{3} 4$ The hapten induced model of colonic inflammation in which 2,4,6-trinitrobenzene sulfonic acid (TNBS) is delivered intrarectally is widely used to test potential therapeutic agents. ${ }^{4}$ In this model, intestinal inflammation results from covalent binding of the haptenising agent to autologous host proteins with subsequent stimulation of a delayed-type hypersensitivity to TNBS modified self antigens. In both CD and TNBS induced colitis, activated Thl cells promote an exaggerated macrophage and neutrophil infiltration and activation, giving rise to a prolonged severe transmural inflamed intestinal mucosa, characterised by uncontrolled production of inflammatory cytokines and chemokines. ${ }^{4}$

Inflammatory mediators such as cytokines (that is, interleukin (IL)-12, interferon $\gamma$ (IFN- $\gamma$ ) and tumour necrosis factor $\alpha$ (TNF- $\alpha)$ ) and nitric oxide (free radical), produced by infiltrating cells and resident macrophages, play a critical role in colonic tissue destruction. Available therapies for CD based on immunosuppressive agents are not entirely effective, are non-specific, and have multiple adverse side effects, and in most cases surgical resection is the ultimate alternative. ${ }^{2}$ This illustrates the need for novel therapeutic approaches that specifically modulate both components of the disease-that is, the inflammatory and Thl driven responses.
Urocortin 1 (UCN) and adrenomedullin (AM) are two recently discovered neuropeptides that are structurally related to corticotrophin releasing factor (CRF) and calcitonin gene related peptide, respectively. ${ }^{5}$ Although UCN and AM were initially known for their cardiovascular protective activities, ${ }^{67}$ several lines of evidence suggest that they could act as endogenous immunomodulatory factors, with predominant anti-inflammatory effects. UCN and AM and their receptors have been detected in several immune cells, including macrophages/monocytes and T cells, in lymphoid organs, and in the gastrointestinal tract, and their expression increases under inflammatory conditions. ${ }^{5-15}$ UCN has been reported to inhibit experimental autoimmune encephalomyelitis, ${ }^{16}$ endotoxin induced TNF- $\alpha$ production by macrophages, ${ }^{17}$ lethal endotoxic shock, ${ }^{18}$ and skin oedema induced by thermal injury. ${ }^{19}$ In addition, UCN present in the mucosa of patients suffering from Helicobacter pylori induced gastritis increases during the stage of eradication and amelioration of inflammation, while in patients resistant to treatment, levels remain low. ${ }^{20}$ In contrast, AM inhibits TNF- $\alpha$ production by activated macrophages and prevents lethal endotoxaemia (unpublished data). ${ }^{13} 182122$

Based on their potential anti-inflammatory actions, we have investigated the potential therapeutic effect of UCN and AM in a murine model of TNBS induced colitis. We report that treatment with UCN or AM significantly reduces the

Abbreviations: AM, adrenomedullin; CD, Crohn's disease; CRF, corticotrophin releasing factor; IL, interleukin; IFN- $\gamma$, interferon $\gamma ; \mathrm{IP}$, inducible protein; $L P M C$, lamina propria mononuclear cells; $\mathrm{mAb}$, monoclonal antibody; MCP, monocyte chemoattractant protein; MIF, macrophage migration inhibitory factor; MIP, macrophage inflammatory protein; MLN, mesenteric lymph nodes; MPO, myeloperoxidase; PMA, phorbol myristate acetate; SAA, serum amyloid A; TGF- $\beta 1$, transforming growth factor $\beta 1$; TNBS, 2,4,6-trinitrobenzene sulfonic acid; TNF- $\alpha$, tumour necrosis factor $\alpha$; Treg, regulatory T cells; UCN, urocortin 
clinical symptoms and pathology by downregulating both inflammatory and Thl mediated immune responses.

\section{MATERIALS AND METHODS \\ Induction of colitis and study design}

Colitis was induced in Balb/c and SJL mice (6-8 weeks old; Jackson Laboratories, Bar Harbor, Maine, USA), as previously described. ${ }^{23}$ In brief, mice were lightly anaesthetised with halothane, and a $3.5 \mathrm{~F}$ catheter was inserted intrarectally $4 \mathrm{~cm}$ from the anus. To induce colitis, TNBS $(3 \mathrm{mg}$; Sigma Chemical Co, St Louis, Missouri, USA) dissolved in 50\% ethanol (to break the intestinal epithelial barrier) was administered into the lumen via a catheter filled from a $1 \mathrm{ml}$ syringe. Control mice received 50\% ethanol alone $(100 \mu \mathrm{l})$. Animals were treated intraperitoneally with medium or with different concentrations of urocortin 1 (UCN $0.05-2.0 \mathrm{nmol} / \mathrm{mouse} ; 10-350 \mu \mathrm{g} / \mathrm{kg})$ or AM $(0.05-2.0 \mathrm{nmol} /$ mouse; $\quad 10-450 \mu \mathrm{g} / \mathrm{kg}$ ) (American Peptides Company, Sunnyale, California, USA) 12 hours after TNBS instillation. To study the therapeutic effect of delayed administration of UCN and AM on established colitis, UCN or AM ( $2 \mathrm{nmol} /$ mouse) was injected intraperitoneally every day for three consecutive days starting six days after TNBS administration. To study the effect on disease recurrence, $1.5 \mathrm{mg}$ of TNBS were administered on days 0 and 9, and UCN or AM ( $2 \mathrm{nmol} /$ mouse) were injected intraperitoneally 12 hours after the first TNBS infusion.

Animals were monitored daily for appearance of diarrhoea, loss of body weight, and survival. Some animals were sacrificed at the peak of the disease (day 3), blood samples were collected by cardiac puncture, and a segment of the colon $(7 \mathrm{~cm}$ long) was excised for evaluation of macroscopic damage and weighed. Tissue segments were immediately frozen in liquid nitrogen for histological and immunohistological studies, protein extraction and cytokine determination, myeloperoxidase (MPO) activity measurement, and total RNA extraction. In another set of experiments, colon segments were used for isolation of lamina propria mononuclear cells (LPMC). All experiments were performed according to the Institutional Guidelines for the Care and Use of Laboratory Animals in Research and the approval of the local ethic committee in the CSIC.

\section{Macroscopic and microscopic damage evaluation}

Colons were examined under a dissecting microscope $(\times 5)$ and graded for macroscopic lesions on a scale from 0 to 10 based on criteria reflecting inflammation, such as hyperaemia, thickening of the bowel, and extent of ulceration. ${ }^{24}$ Scores for stool consistency and rectal bleeding were assessed according to previously published procedures. ${ }^{25}$ For histopathological analysis, colons were fixed, sectioned, and stained with haematoxylin-eosin, and inflammation was graded from 0 to 4 as follows, in a blinded fashion: 0 , no signs of inflammation; 1, low leucocyte infiltration; 2, moderate leucocyte infiltration; 3 , high leucocyte infiltration, moderate fibrosis, high vascular density, thickening of the colon wall, moderate goblet cell loss, and focal loss of crypts; and 4, transmural infiltrations, massive loss of goblet cell, extensive fibrosis, and diffuse loss of crypts. For immunohistological analysis, sections were stained with $5 \mu \mathrm{g} / \mathrm{ml}$ FITC and PE labelled anti-CD4, anti-CD1 lb, or anti-TNF- $\alpha$ monoclonal antibodies (mAbs) and examined by fluorescent microscopy (Leica, Wetzlar, Germany).

\section{Cytokine determination}

For cytokine determination in colon mucosa, protein extracts were obtained by homogenisation of colonic segments (0.5 mg tissue/ml) in $50 \mathrm{mM}$ Tris $\mathrm{HCl}, \mathrm{pH}$ 7.4, $0.5 \mathrm{mM}$ DTT, and $10 \mu \mathrm{g} / \mathrm{ml}$ of a cocktail of proteinase inhibitors containing phenylmethylsulfonyl fluoride, pepstatin, and leupeptin (Sigma). Samples were centrifuged at $30000 \mathrm{~g}$ for $20 \mathrm{~min}$ utes and stored at $-80^{\circ} \mathrm{C}$ until cytokine determination. Cytokine and chemokine levels in serum, colonic protein extracts, and culture supernatants were determined by a specific sandwich ELISA using capture/biotinylated detection Abs from BD Pharmingen (San Diego, California, USA) and Preprotech (Rocky Hill, New Jersey, USA) according to the manufacture's recommendations. Release of total transforming growth factor $\beta 1$ (TGF- $\beta 1$ ) (active+latent) was determined by ELISA (BD Pharmingen) after acidification with $0.1 \mathrm{M} \mathrm{HCl}$ and neutralisation of culture supernatants. Serum amyloid A (SAA) levels were determined in serum samples by a murine ELISA kit (Tridelta Development, New Jersey, USA). For intracellular analysis of cytokines in stimulated LPMC and mesenteric lymph node (MLN) cells, $10^{6}$ cells $/ \mathrm{ml}$ were collected and stimulated with phorbol myristate acetate (PMA $1 \mathrm{ng} / \mathrm{ml})$ plus ionomycin $(20 \mathrm{ng} / \mathrm{ml})$ for eight hours in the presence of monensin. Cells were stained with PerCPanti-CD4 mAbs, fixed/saponin permeabilised with Cytofix/ Cytoperm, stained with FITC and PE conjugated anticytokine specific mAbs (BD Pharmingen), and analysed a FACScalibur flow cytometer (BD Pharmingen). In order to distinguish between monocyte/macrophage and T cell sources, intracellular cytokine analysis was done exclusively in the PerCP labelled CD4 T cell population.

\section{MPO assay}

Neutrophil infiltration in the colon was monitored by measuring MPO activity using a method reported previously. ${ }^{26}$

\section{Isolation and culture of LPMC and mesenteric lymph node cells (MLN)}

LPMC were isolated from freshly obtained colonic specimens using a previously described technique. ${ }^{23}$ MLN cells were enriched in T cells by incubating MLN cells in Petri dishes for two hours at $37^{\circ} \mathrm{C}$ to remove non-adherent cells. LPMC and MLN cells were incubated in complete medium (RPMI-1640 supplemented with $100 \mathrm{U} / \mathrm{ml}$ penicillin/streptomycin, $2 \mathrm{mM}$ L-glutamine, $50 \mu \mathrm{M}$ 2-mercaptoethanol, and $10 \%$ heat inactivated fetal calf serum) at $10^{6}$ cells $/ \mathrm{ml}$, in the absence (unstimulated) or presence of PMA $(10 \mathrm{ng} / \mathrm{ml})$ and Con A $\left(2.5 \mu \mathrm{g} / \mathrm{ml}\right.$ ). Cell proliferation (expressed as $\mathrm{A}_{450}$ ) was evaluated in 96 well microtitre plates for 96 hours using a cell proliferation assay (BrdU) from Roche Diagnostics $\mathrm{GmbH}$ (Mannheim, Germany). Cytokine/chemokine production in culture supernatants was determined after 48 hours of culture, as described above. For TGF- $\beta 1$ production, cells were cultured additionally for 24 hours in serum free medium. Intracellular cytokine content was determined in stimulated cells, as described above.

\section{Gene expression analysis}

Total RNA was isolated from colonic specimens using Ultraspec RNA reagent (Biotecx, Houston, Texas, USA) and mRNA expression of a variety of chemokines, chemokine receptors, cytokines, enzymes, and leucocyte markers was quantified using a GEArray focused DNA microarray for 96 inflammatory and autoimmune agents (Superarray Bioscience Corporation; Frederick, Maryland, USA) following the manufacturer's recommendations. Sample to sample variation in RNA loading was controlled by comparison with the housekeeping gene G3PDH.

\section{Analysis of Foxp 3 expression by flow cytometry} MLN cells $\left(10^{6}\right.$ cells) were isolated and stained with PerCPanti-CD4 and FITC-anti-CD25 mAbs (BD Pharmingen) for one hour at $4^{\circ} \mathrm{C}$, washed, fixed/saponin permeabilised with Cytofix/Cytoperm, stained with $0.5 \mu \mathrm{g} /$ sample of PE conjugated 
A
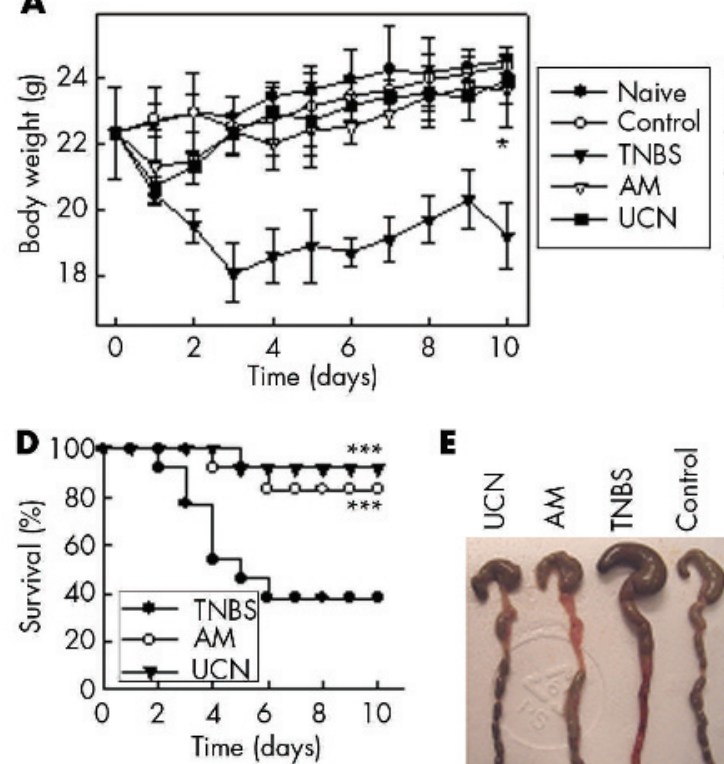

$$
\mathbf{F}
$$
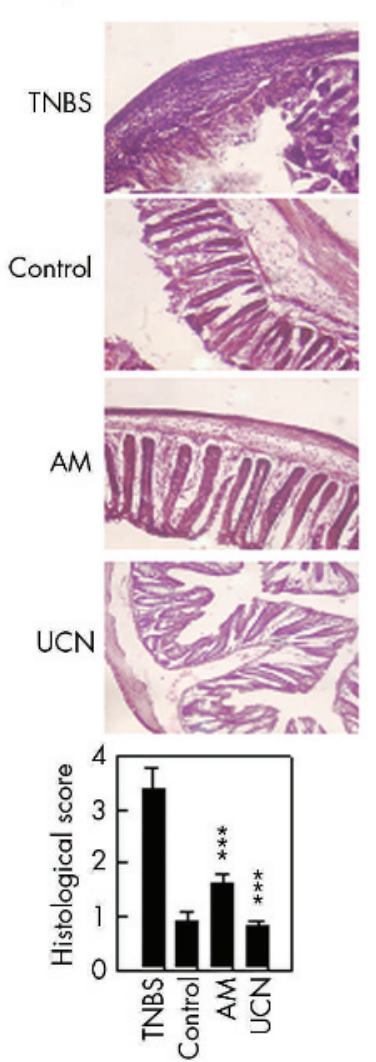

E

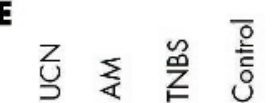

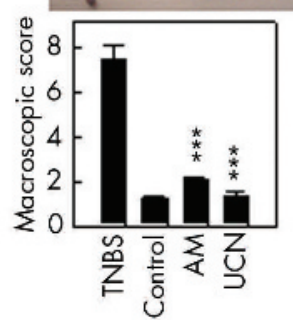

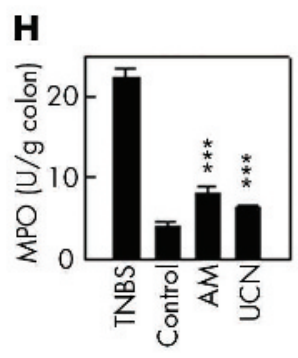

B

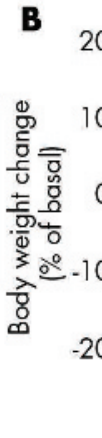

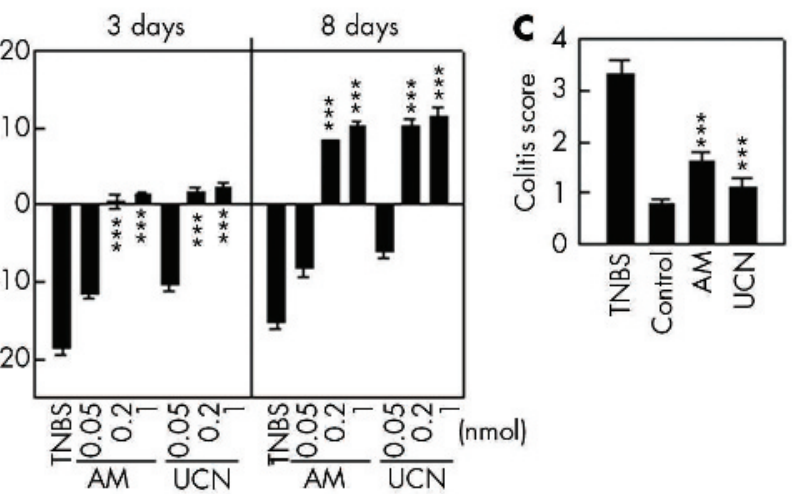

$$
\mathbf{G}
$$

CDIlb

TNF- $\alpha$

CD4
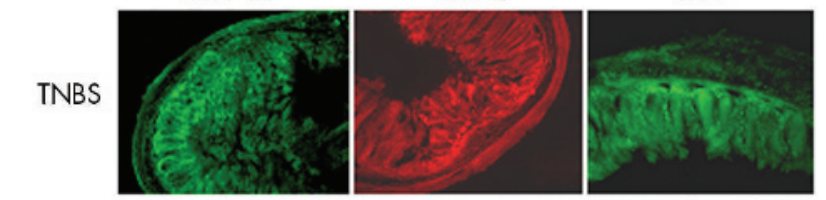

Control
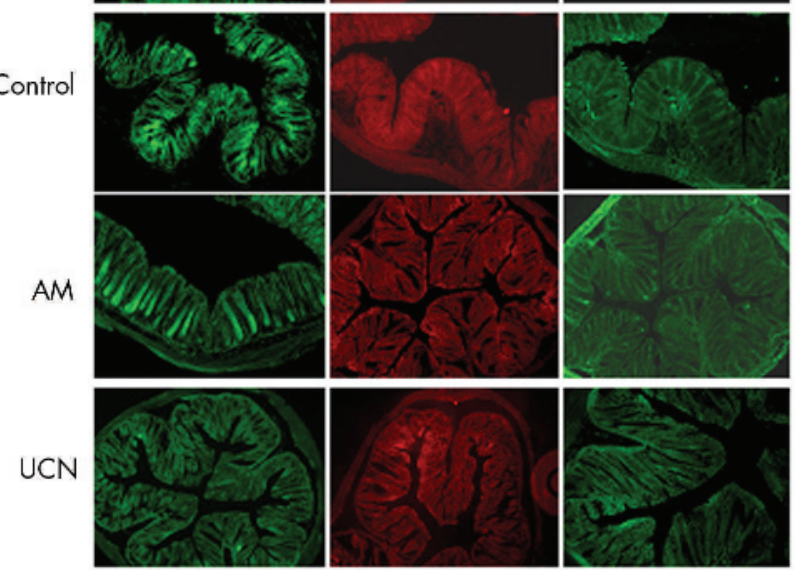

Figure 1 Treatment with urocortin (UCN) or adrenomedullin (AM) protects against 2,4,6-trinitrobenzene sulfonic acid (TNBS) colitis development. Colitis was induced by intracolonic administration of TNBS (3 mg/mouse) in $50 \%$ ethanol. Ballb/c mice were treated intraperitoneally with AM or UCN $(2 \mathrm{nmol} /$ mouse, or different doses in B) 12 hours after TNBS injection. Mice treated with $50 \%$ ethanol were used as controls. Clinical evolution and severity were monitored by body weight changes (A, B), colitis score (C), and survival (D). Macroscopic damage score was determined three days after TNBS administration (E). Histopathological analysis was determined in haematoxylin-eosin stained sections of colons at day 3 of disease (F) $(\times 200)$. Inflammatory infiltrates in the colons (day 3 ) were phenotypically characterised by immunostaining against CD1 $1 \mathrm{~b}$ (monocyte/macrophage and neutrophils), tumour necrosis factor $\alpha$ (TNF- $\alpha)$ producing cells, or CD4 T cells $(G)(\times 200)$. Colonic myeloperoxidase $(M P O)$ activity was determined in the acute phase of the disease (day 3$)(H) . n=12-18$ mice/group. ${ }^{* *} p<0.001$ versus TNBS treated mice. 

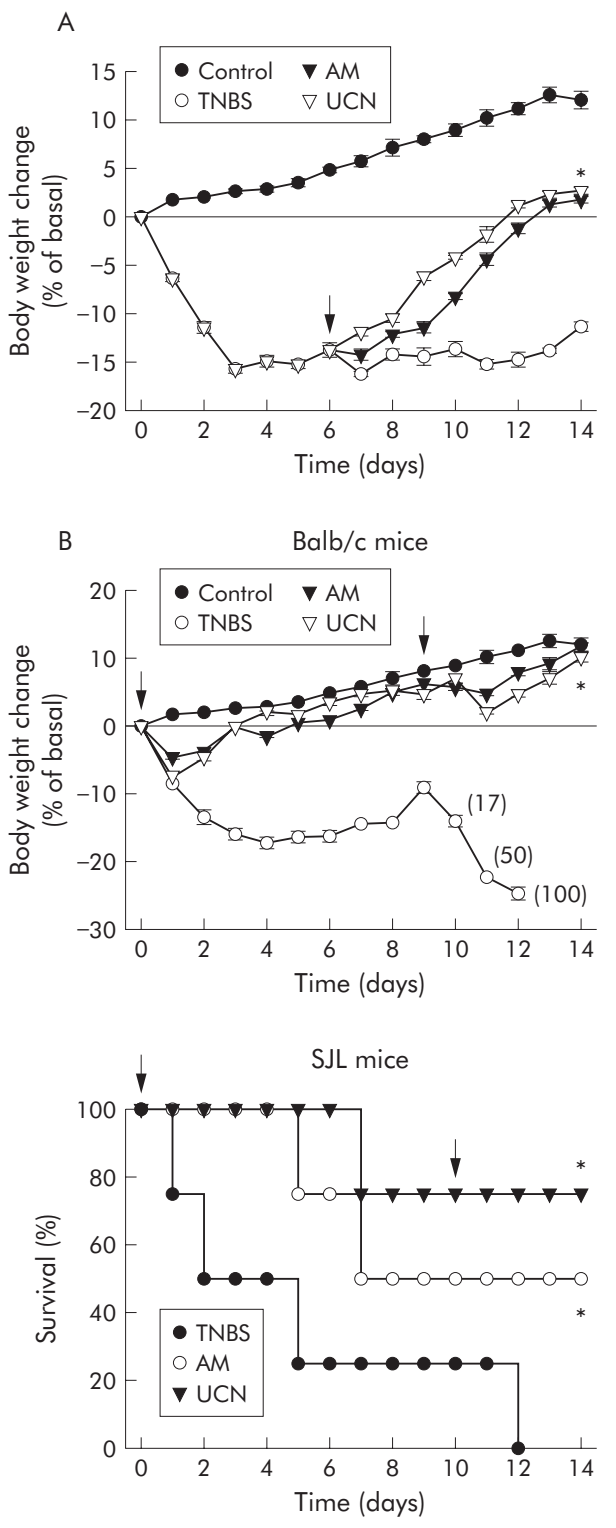

Figure 2 Urocortin (UCN) and adrenomedullin (AM) abrogate established colitis and reduce disease recurrence. (A) Established colitis. Colitis was induced by intracolonic administration of 2,4,6trinitrobenzene sulfonic acid (TNBS $1.5 \mathrm{mg} / \mathrm{mouse}$ ) on days 0 and 6 . Mice were treated daily for three consecutive days with UCN or AM ( $2 \mathrm{nmol} /$ mouse) starting six days after TNBS administration (arrow). Disease progression was assessed by body weight loss and survival percentage. $n=8$ mice/group. (B) Disease recurrence. Colitis was induced in Balb/c and SJL mice by intracolonic administration of TNBS ( $3 \mathrm{mg} / \mathrm{mouse}$ ) on days 0 and 9 (arrows). Mice were treated intraperitoneally with UCN or AM ( $2 \mathrm{nmol} /$ mouse) 12 hours after TNBS injection. Controls were given a second injection of ethanol on day 9. Disease progression was assessed by body weight loss and survival percentage. Numbers in parentheses represent daily mortality percentage after the second TNBS infusion. $n=8-10$ mice/group. ${ }^{*} p<0.001$ versus TNBS treated mice.

anti-Foxp3 mAb (eBioscience San Diego, California, USA), and analysed on a FACScalibur flow cytometer.

\section{Data analysis}

All values are expressed as mean (SD) of mice/experiment. Differences between groups were analysed using the MannWhitney U test and, if appropriate, by the Kruskal-Wallis ANOVA test. Survival curves were analysed by the KaplanMeyer log rank test. Changes in body weight were compared using the Wilcoxon matched pair signed rank test.

\section{RESULTS}

\section{Treatment with UCN or AM protects against TNBS} colitis development

We investigated the potential therapeutic action of AM and UCN in the TNBS model of colitis. Balb/c mice subjected to intrarectal administration of TNBS in 50\% ethanol developed a severe illness characterised by bloody diarrhoea, rectal prolapse, pancolitis accompanied by extensive wasting syndrome, and a profound and sustained weight loss resulting in a mortality rate of $60 \%$ (fig $1 \mathrm{~A}-\mathrm{D}$ ). Mice treated with AM or UCN 12 hours after TNBS instillation rapidly recovered lost body weight, improved their wasting disease, and had a healthy appearance, with a survival rate greater than $80 \%$, similar to control mice treated with $50 \%$ ethanol alone (fig 1A-D). The therapeutic effects of AM and UCN were dose dependent, showing maximal effects at doses of $0.2-2 \mathrm{nmol}(45-450 \mu \mathrm{g} / \mathrm{kg}$ ) (fig lB). Macroscopic examination of colons obtained three and seven days after colitis induction showed striking hyperaemia, inflammation, and necrosis compared with control animals that showed only slight inflammation (fig 1E). In contrast, the colons of AM/ UCN treated mice showed no signs of macroscopic inflammation (fig lE).

Histological examination of the distal colon of mice given TNBS showed transmural inflammation involving all layers of the bowel wall with a marked increase in the thickness of the muscular layer, adherence to surrounding tissues, patchy ulceration, epithelial cell loss, pronounced depletion of mucin producing goblet cells, reduction of the density of tubular glands, and focal loss of crypts (fig lF). Inflammatory cell infiltrates consisted of macrophages, lymphocytes, and neutrophils in the lamina propria (fig IF), along with enlargements of lymphoid follicles in the colon (not shown). Immunohistological analysis revealed CD4 T cells, TNF- $\alpha$ producing cells, and CDI lb cells-that is, granulocytes and macrophages (fig lG). Neutrophil infiltration correlated with increased colonic MPO activity (fig $1 \mathrm{H}$ ). When mice were treated with UCN or AM, striking improvement of these macroscopic and histological signs became apparent, with a significant reduction in inflammatory activity and neutrophil infiltration (fig $1 \mathrm{~F}-\mathrm{H}$ ).

We next investigated whether UCN/AM treatment would be effective during the later phases of the disease with colitis fully established. Administration of AM or UCN on three consecutive days starting six days after onset of disease rapidly reversed lost body weight (fig $2 \mathrm{~A}$ ). In addition, we examined whether AM and UNC were able to prevent disease recurrence. TNBS treated mice re-exposed on day 9 to a second dose of TNBS, rapidly died (100\% mortality) due to severe colitis and body weight loss. Mice receiving a unique dose of AM or UCN 12 hours after the initial colitis induction survived and did not suffer disease recurrence after a second administration of TNBS (fig 2B). The therapeutic effect of both peptides was confirmed in SJL mice, a murine strain more susceptible to TNBS induced colitis (fig 2B).

\section{Treatment with UCN or AM reduces systemic and mucosal inflammatory responses in mice with TNBS induced colitis}

We next evaluated the effect of UCN and AM on the production of inflammatory mediators that are mechanistically linked to TNBS induced colitis. Treatment with UCN or AM dramatically reduced protein and mRNA expression of inflammatory cytokines (TNF- $\alpha$, IFN- $\gamma$, IL-6, IL- $1 \alpha$, IL-1 $\beta$, IL-12, IL-18, IL-17, IL-15, and macrophage migration inhibitory factor (MIF)), chemokines (Rantes, macrophage inflammatory protein (MIP)-1 $\alpha$, MIP-1 $\beta$, MIP-3 $\beta$, monocyte chemoattractant protein (MCP)-1, MCP-3, inducible protein (IP)-10, and MIP-2) and chemokine receptors (CCR-1, 
A

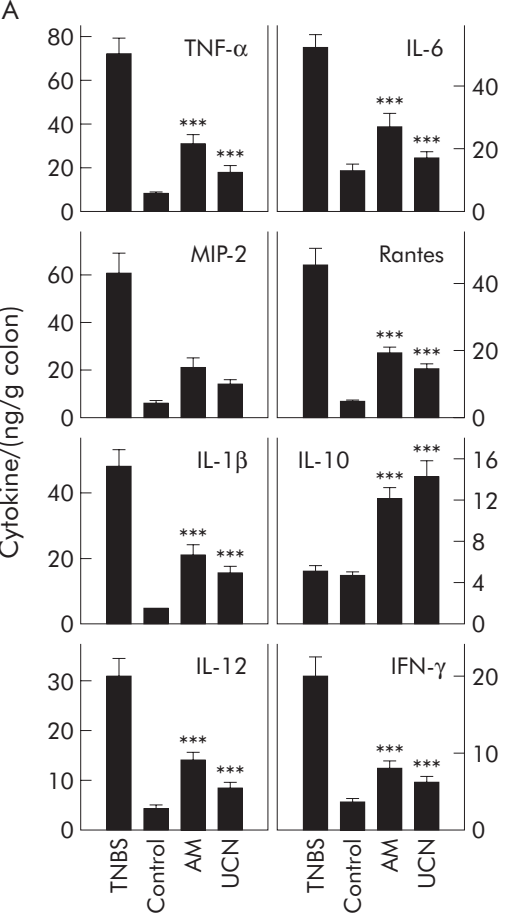

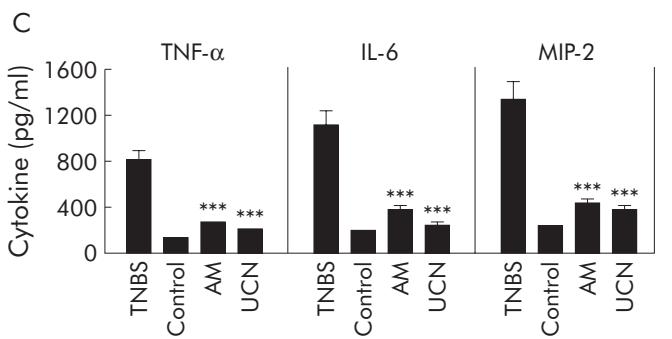

Figure 3 Urocortin (UCN) and adrenomedullin (AM) decrease mucosal and systemic inflammatory responses in mice with 2,4,6-trinitrobenzene sulfonic acid (TNBS) induced colitis. Colitis was induced by intracolonic administration of TNBS. Mice were treated intraperitoneally with AM or UCN

( $2 \mathrm{nmol} /$ mouse) 12 hours after TNBS injection. Mice treated with ethanol alone were used as controls. At the peak of the disease (day 3 ), serum was collected, protein extracts and total RNA were obtained from colons, and lamina propria mononuclear cells (LPMN) were isolated. (A) Cytokine/ chemokine content in protein extracts was determined by ELISA. $n=5-6$ mice/group. (B) Gene expression of several inflammatory/autoimmune mediators was determined using a microarray. Results are representative of two separate experiments $(n=4-5$ mice/group). (C) Isolated LPMC were cultured in vitro for 24 hours and cytokine/chemokine content in the supernatants was determined by ELISA. $\mathrm{n}=5-6$ mice/group. (D) Cytokine/ chemokine and serum amyloid $A$ (SAA) content was determined in sera by ELISA. $\mathrm{n}=5-6$ mice/group.
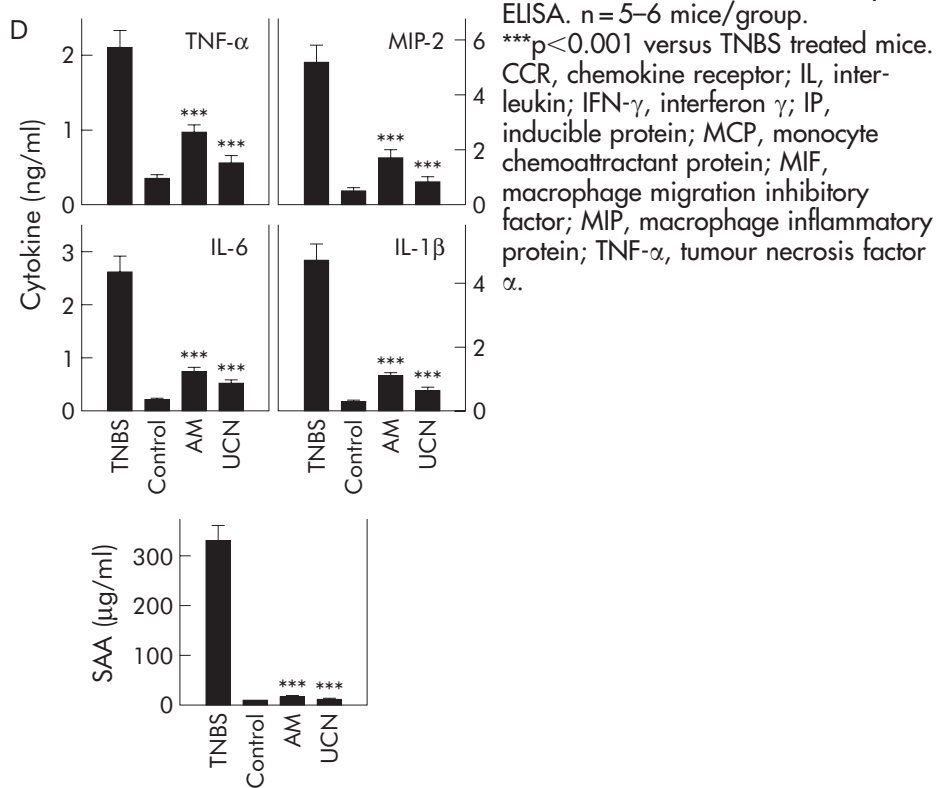

CCR-2, CCR-3, CCR-5, and CCR-7) in the mucosa of colitic mice (fig 3A, B). In addition, colons of AM/UCN treated mice showed increased levels of the anti-inflammatory cytokine IL-10 and chemokine receptors CCR-4 and CCR-8 (fig 3A, B). The decrease in inflammatory mediators could be a consequence of the diminished infiltration of inflammatory cells in the colonic mucosa in AM/UCN treated colitic mice. However, LPMC isolated from mice treated with AM or UCN produced lower levels of proinflammatory factors (TNF- $\alpha$, IL6 , and MIP-2) on in vitro culture in comparison with TNBS mice (fig 3C). This suggests that, in addition to the reduction in inflammatory infiltration, UCN/AM administration deactivates the inflammatory response in the colonic mucosa. The broad anti-inflammatory activity of both peptides in the colon was accompanied by downregulation of the systemic inflammatory response implicated in colonic inflammation (fig 3D). UCN/AM treatment decreased TNBS induced serum levels of the proinflammatory cytokines TNF- $\alpha$, IL-1 $\beta$, IL-6, and MIP-2, and of SAA, an hepatic acute phase protein involved in tissue damage in inflammatory conditions. ${ }^{27}$

\section{UCN and AM suppress Th 1 cytokine response and stimulate IL-10/TGF- $\beta 1$ production in TNBS induced colitis}

Although macrophages and neutrophils are the major sources of inflammatory mediators, CD4 T cells also play a key role in the initiation and perpetuation of CD by producing IFN- $\gamma$, a potent inducer of the inflammatory response. ${ }^{4}$ Although important discrepancies exist in the literature, ${ }^{28}{ }^{29}$ the bias towards Thl cytokines (mainly IFN- $\gamma$ and TNF- $\alpha$ ) seems to be crucial in the establishment of chronic inflammation. Therefore, we determined the effect of treatment of TNBS induced colitis with UCN or AM on the ability of LPMC and draining MLN to produce IFN- $\gamma$ and proliferate in vitro. LPMC and MLN cells obtained from TNBS treated mice proliferated more and produced significantly more IFN- $\gamma$ 

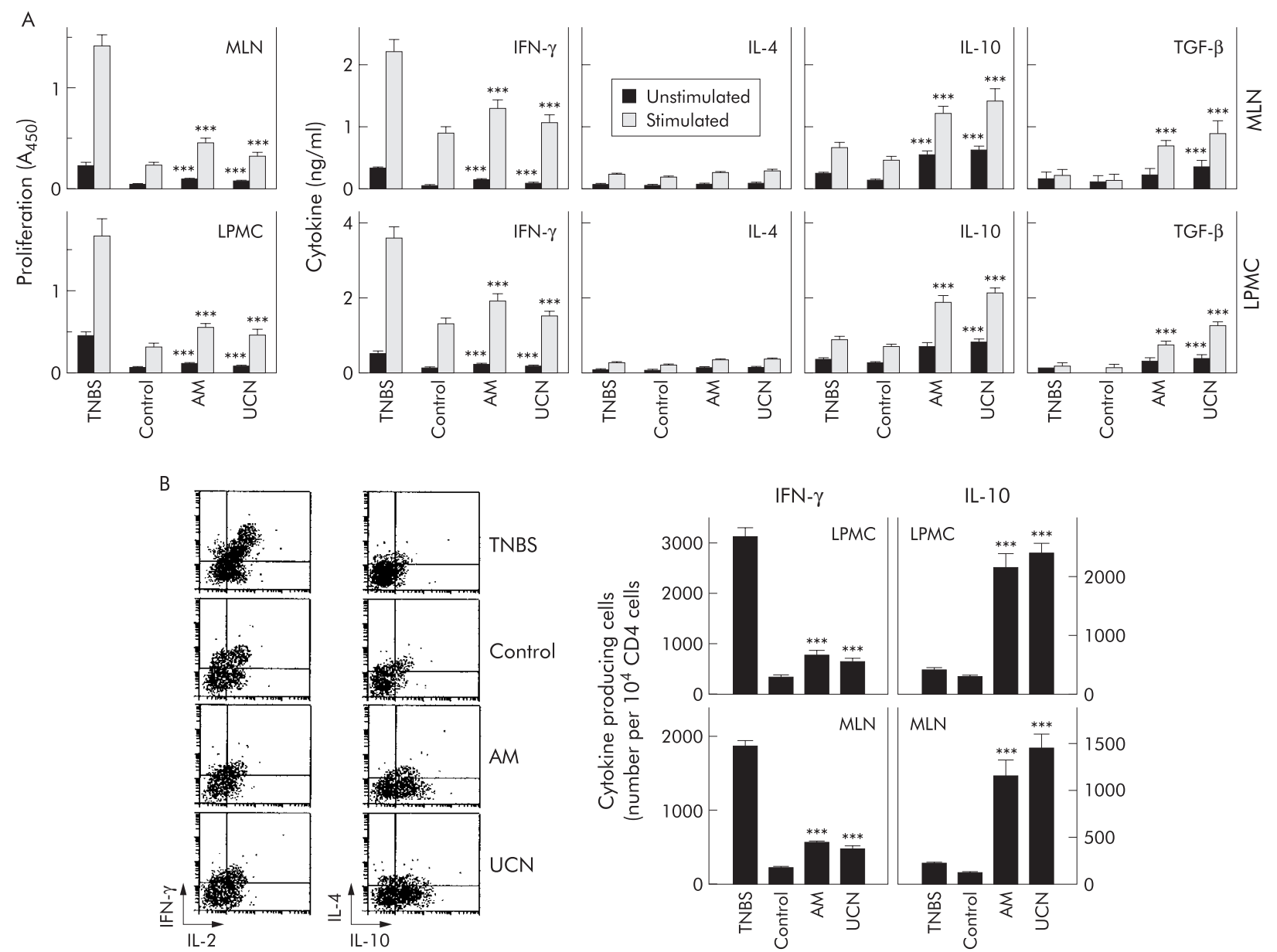

Figure 4 Urocortin (UCN) and adrenomedullin (AM) suppress Th1 cytokine response and stimulate interleukin (IL)-10 production in 2,4,6-trinitrobenzene sulfonic acid (TNBS) induced colitis. Colitis was induced by intracolonic administration of TNBS and mice were treated intraperitoneally with AM or UCN ( $2 \mathrm{nmol} / \mathrm{mouse}) 12$ hours after TNBS injection. Mice treated with ethanol alone were used as controls. Mesenteric lymph node cells (MLN) and lamina propria mononuclear cells (LPMN) were isolated from the different experimental groups at the peak of the disease (day 3), and cultured with medium alone (unstimulated) or with phorbol myristate acetate plus concanavalin A (stimulated). (A) Proliferative response was determined after four days of culture, as described in the methods. Cytokine content in the supernatants was determined after 48 hours of culture by ELISA. (B)

Stimulated lymphocytes were analysed for CD4 and intracellular cytokine expression by flow cytometry. Double staining for interferon $\gamma$ (IFN- $\gamma$ )/IL-2 or IL-4/IL-10 expression was performed in gated CD4 T cells. The number of IFN- $\gamma$ and IL-10 expressing T cells relative to $10^{4}$ CD4 T cells was determined (right panels). Data shown represent pooled values from two independent experiments ( $n=5-6$ mice/group/experiment). ${ }^{* * *} p<0.001$ versus TNBS treated mice. TGF- $\beta 1$, transforming growth factor $\beta 1$.

than control (ethanol treated) mice, and in vitro activation of these cells caused further cell expansion and increased amounts of IFN- $\gamma$ (fig 4A, B). In contrast, LPMC and MLN cells isolated from colitic animals treated with AM or UCN proliferated less and produced significantly lower amounts of IFN- $\gamma$ than cells isolated from TNBS treated mice, even after potent $\mathrm{T}$ cell stimulation with PMA/concanavalin A (fig 4A, B). In addition, production of the regulatory cytokines IL-10 and TGF- $\beta 1$ was significantly increased in LPMC and MLN cells obtained from UCN/AM treated mice, specially on in vitro activation; the Th2-type cytokine IL-4 was not significantly affected (fig 4B). Thus treatment with UCN or AM resulted in decreased Thl cytokine production in vivo, and abrogated the responsiveness of LPMC and draining MLN cells to subsequent in vitro stimulation.

Decreased IFN $-\gamma$ production and proliferation of LPMC and MLN cells was specific to cells residing in the lamina propria environment or draining lymph nodes, as splenocytes from UCN/AM treated and untreated TNBS mice equally proliferated and produced IFN- $\gamma$ on stimulation (not shown). Given that the decrease in IFN- $\gamma$ production induced by UCN/AM treatment could be a consequence of either downregulation of IFN- $\gamma$ release or inhibition of Thl cell differentiation, and that production of IL-10 could be due to macrophages and CD4 T cells, we determined intracellular expression of these cytokines by flow cytometry in sorted CD4 T cells. AM and UCN significantly decreased the number of IL- $2 / \mathrm{IFN}-\gamma$ producing Thl cells and increased the number of IL-10 producing CD4 T cells in LPMN and MLN (fig 4B, right panels). Thus UCN/AM administration to colitic mice regulates the generation/differentiation of autoreactive/inflammatory Thl cells and presumably regulatory IL-10/TGF- $\beta$ secreting T cells.

Although IL-10 and TGF- $\beta$ are factors involved in the function of regulatory $\mathrm{T}$ cells, they are not exclusive markers of $\mathrm{T}$ cells. Therefore, in order to confirm whether treatment with UCN and AM induces the appearance of regulatory $\mathrm{T}$ cells on colitic mice, we determined the percentage of $\mathrm{CD}^{+} \mathrm{CD} 25^{+} \mathrm{Foxp}^{+} \mathrm{T}$ cells in MLN, a phenotype that closely identifies regulatory $\mathrm{T}$ cells. Administration of AM or UCN significantly increased the presence of $\mathrm{CD}^{+} \mathrm{CD} 25^{+} \mathrm{Foxp}^{+} \mathrm{T}$ cells in MLN (fig 5). Interestingly, the increased number of $\mathrm{CD}^{+} \mathrm{CD} 25^{+} \mathrm{Foxp}^{+} \mathrm{T}$ cells induced by both neuropeptides persisted for a long period of time, even when a second TNBS dose was given (fig 5). 


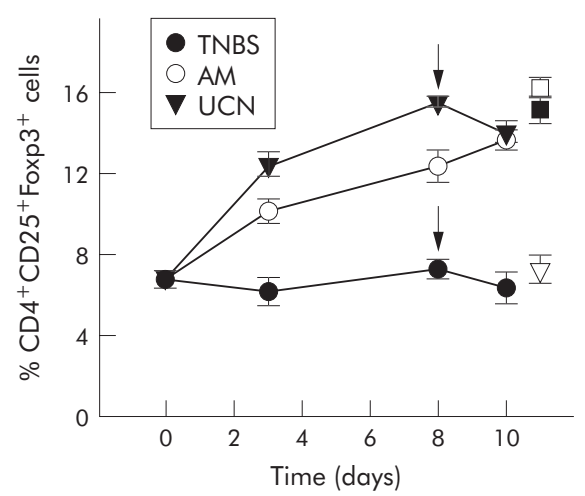

Figure 5 Urocortin (UCN) and adrenomedullin (AM) induce the appearance of $\mathrm{CD} 4^{+} \mathrm{CD} 25^{+}$Foxp $3^{+} \mathrm{T}$ cells in 2,4,6-trinitrobenzene sulfonic acid (TNBS) induced colitis. Colitis was induced by intracolonic administration of TNBS. Mice were treated intraperitoneally with UCN or AM ( $2 \mathrm{nmol} /$ mouse), 12 hours after TNBS injection. Mesenteric lymph node cells were isolated from the different experimental groups at different times after TNBS infusion and the percentage of $\mathrm{CD} 4^{+} \mathrm{CD} 25^{+} \mathrm{Foxp}^{+}$cells was determined by flow cytometry. Alternatively, a second TNBS dose ( $3 \mathrm{mg} / \mathrm{mouse})$ was given on day 8 (arrow) to untreated mice (open triangle) and mice treated with AM (closed square) or UCN (open square) and the percentage of $\mathrm{CD} 4^{+} \mathrm{CD} 25^{+}$Foxp $3^{+}$cells was determined three days later. $\mathrm{n}=5$ mice/ group.

\section{DISCUSSION}

The present study has shown that UCN and AM are two potent anti-inflammatory agents that provide highly effective treatment for TNBS induced colitis, a murine experimental model of CD. A single injection of UCN or AM $(450 \mu \mathrm{g} / \mathrm{kg})$ at the onset of the disease ameliorated the clinical and histopathological severity of the wasting disease, abrogating body weight loss, diarrhoea, and intestinal inflammation, and reduced the high mortality caused by this syndrome. From a therapeutic point of view, it is extremely important to take in account that delayed treatment with UCN or AM was able to reduce colitis severity in animals with established disease, and that an initial treatment with UCN or AM prevented recurrence of the disease in mice given a second dose of TNBS.

There are several potential mechanisms by which therapy with UCN and AM can modulate the effector phase of TNBS colitis. CD and TNBS induced colitis are characterised by transmural inflammation of the colon due to an IL-12 driven, Thl cell mediated response to TNBS haptenated colonic proteins and/or crossreactive luminal antigens. ${ }^{4}$ During the effector phase of bowel inflammation, both innate and acquired immune responses overlap, and multiple inflammatory mediators are involved. UCN and AM strongly reduced mucosal inflammation by downregulating the production of a wide panel of mediators involved in the local and systemic inflammatory response. Chemokines are responsible for mucosal infiltration and activation of various leucocyte populations which contribute to colitis development. ${ }^{30}$ The fact that treatment with UCN or AM reduced the production of a plethora of chemokines could partially explain the absence of inflammatory infiltrates in the colonic mucosa of UCN/AM treated mice, being especially relevant for chemokines such as MIP-2 (chemotactic for neutrophils), IP-10 (for Thl cells), and Rantes/MIPl $\alpha$ (for macrophages and T cells), all involved in CD pathogenesis. ${ }^{30}$

In addition to regulation of cell recruitment to the lamina propria during colitis, UCN and AM also regulate inflammatory cell activation and cytokine production. Thus both peptides downregulated the production of the proinflammatory/cytotoxic cytokines TNF- $\alpha$, IFN- $\gamma$, IL-6, IL-1 $\beta$, IL-12,
IL-15, IL-17, IL-18, and MIF by mucosal immune cells, and increased levels of the anti-inflammatory cytokine IL-10. The decrease in inflammatory mediators could be a consequence of diminished infiltration of inflammatory cells into the colonic mucosa in UCN/AM treated TNBS mice. However, the fact that LPMC isolated from mice treated with UCN or AM produced lower levels of proinflammatory factors on in vitro activation argues against this hypothesis. This suggests that, in addition to the reduction in inflammatory infiltration, UCN/AM administration deactivates the inflammatory response. Several studies have demonstrated that UCN and AM downregulate the production of inflammatory mediators by activated macrophages. Therefore, it is plausible that deactivation of resident and infiltrating mucosal macrophages could be a major mechanism involved in the antiinflammatory action of UCN and AM in colitis.

The local anti-inflammatory action of AM and UCN was also evident systemically. Of special consideration is the reduction in SAA, an acute phase protein used for clinical monitoring of $\mathrm{CD}^{31}$ which has been associated with tissue damage in several inflammatory conditions ${ }^{27}$ although its precise role in intestinal inflammation is not known. At this point, the obligatory question is: how do AM and UCN regulate such a wide spectrum of inflammatory mediators? The answer may be found in the fact that the cAMP/protein kinase A pathway is the major intracellular signal involved in most of the effects of AM and UCN, including downregulation of inflammatory mediators. ${ }^{17}{ }^{22}$ CAMP inducing agents have been found to be potent anti-inflammatory factors which act by downregulating activation of the transcription factor nuclear factor $\kappa \mathrm{B}$, a factor essential for the transcriptional activation of most of the inflammatory cytokines and chemokines. The effects of AM and UCN on activation of nuclear factor $\mathrm{\kappa B}$ and other transcription factors are currently under investigation in our laboratory.

TNBS induced colitis is also a Thl mediated disease, requiring $\mathrm{T}$ cell activation as one of the initiating events that subsequently leads to macrophage/neutrophil recruitment and activation. ${ }^{4}$ Although in some instances the beneficial effects of Thl-type cytokines (mainly IFN- $\gamma$ ) in systemic and organ specific autoimmune diseases have been described, in most cases detrimental effects have been demonstrated. ${ }^{2328} 29$ In fact, high levels of Thl-type cytokines (for example, IFN- $\gamma$ ) are detected in the colon at the height of disease in both CD and TNBS induced colitis and, conversely, neutralising Thl-type cytokine specific antibodies ameliorates disease progression in a murine model. ${ }^{423}{ }^{28}$ Our results demonstrate that expression of the Thl-type cytokines IFN- $\gamma$ and TNF- $\alpha$ in the colon is downregulated in response to UCN or AM after induction of TNBS colitis. It appears that inhibition of the Thl response is caused by a direct action on LPMC and draining MLN cells as LPMC and MLN cells obtained from UCN/AM treated animals are refractory to Thl cell stimulation.

In contrast with IFN- $\gamma$ and TNF- $\alpha$, UCN and AM increased production of IL-10 in vivo. However, the fact that both peptides increased IL-10, but not IL-4, production, in CD4 T cells from LPMC and MLN, argues against a shift towards Th2 responses. IL-10 has been recognised recently as a signature cytokine for a subset of CD4 T cells that exert regulatory functions. ${ }^{31}$ Active suppression by IL-10 secreting regulatory $\mathrm{T}$ cells (Treg) plays a key role in the control of self antigen reactive $\mathrm{T}$ cells and induction of peripheral tolerance in vivo. ${ }^{32}{ }^{33}$ In fact, deletion or dysfunction of these suppressive cells results in the appearance of multiple autoimmune-inflammatory disorders, especially in the intestine. $^{34-36}$ In addition, UCN/AM treatment increased the production of TGF- $\beta 1$ by activated LPMC and MLN cells, and TGF- $\beta 1$ is another mediator of Treg function. ${ }^{34}{ }^{35}$ Our 
data suggest that both neuropeptides favour the generation/ activation of IL-10/TGF- $\beta$ secreting Treg cells, a finding that correlates with the fact that treatment with AM or UCN increased the percentage of Foxp3 expressing $\mathrm{CD}^{+} \mathrm{CD}^{+} 5^{+} \mathrm{T}$ cells in MLN, a phenotype characteristic of Treg cells. ${ }^{32}{ }^{34}$ In addition, both peptides increased colonic expression of CCR-4 and CCR-8, two chemokine receptors expressed in Treg cells. ${ }^{37}{ }^{38}$ UCN and AM may favour the recruitment of Treg to the inflamed mucosa. However, we did not observed a significant increase in expression of ligands for CCR4 and CCR8 (CCL17 and CCL1) in the colon after UCN/AM treatment. Although the mechanism is still unknown, preliminary experiments indicate that both neuropeptides could induce differentiation of tolerogenic dendritic cells with the capacity to generate IL-10-secreting Treg cells (unpublished results). Maintenance of tolerance by IL-10/ TGF- $\beta$ secreting $\mathrm{T}$ cells could be related to resistance to disease recurrence observed in AM/UCN treated mice, mainly because the number of $\mathrm{CD} 4^{+} \mathrm{CD} 25^{+} \mathrm{Foxp} 3^{+} \mathrm{T}$ cells persisted for a long period and when a second TNBS dose was given.

Improvement in wasting disease seen in mice treated with UCN or AM (approximately 90\%) compares favourably with that achieved with other therapies, such as blocking of IL-12, TNF- $\alpha$, IL-6, or IFN $\gamma$, or treatment with prednisolone or mesalamine, ${ }^{23} 3^{39-42}$ some of which are widely used in treating inflammatory bowel disease patients. The capacity of both peptides to regulate a wide spectrum of inflammatory mediators, in addition to suppression of Thl-type responses and potential generation of Treg cells, might offer a therapeutic advantage over neutralising antibodies directed against a single mediator.

UCN is structurally related to CRF and is an endogenous ligand of CRF receptors. Depending on the site of secretion, both pro- and anti-inflammatory actions have been attributed to CRF. Both physical and psychological stress induce central and peripheral expression of CRF and UCN. Central secretion of CRF activates the hypothalamus-pituitaryadrenal axis that ultimately induces release of corticosteroids, which mediate the anti-inflammatory effects of central CRF. $^{43-45}$ In contrast, peripherally secreted CRF is largely proinflammatory, especially in the gastrointestinal tract. ${ }^{46-48}$ In contrast with CRF, the anti-inflammatory effect of UCN in systemic inflammation and experimental autoimmune encephalomyelitis is corticosteroid independent. ${ }^{17}{ }^{19}$ The present study and other evidence suggest that UCN at inflammation sites may counteract the proinflammatory effects of CRF. ${ }^{47}$ Interestingly, UCN preferentially binds to the CRF receptor type 2 with higher affinity than CRF, and the distribution of UCN, but not of CRF, correlates with the distribution of the CRF receptor type 2 in the periphery. ${ }^{5}$

Of physiological relevance is the observation that expression of UCN and AM is increased in activated inflammatory cells. ${ }^{5-15}$ Although levels of UCN and AM have not yet been measured in CD patients, they are elevated in several inflammatory conditions, including rheumatoid arthritis, endotoxaemia, gastritis, and ulcerative colitis. ${ }^{13} 192049$ Therefore, it is attractive to speculate that the body responds to an exacerbated inflammatory response by increasing the peripheral production of endogenous anti-inflammatory factors, including UCN and AM. Although both peptides have differing actions affecting the cardiovascular system (stress and anxiety), we did not observe any adverse effects of the peptides at the concentrations used in this murine system. The ability of delayed administration of UCN and AM to ameliorate ongoing disease also fulfils an essential prerequisite for any anticolitis agent, as treatment is started after the onset of CD patients.

In summary, we have identified UCN and AM as potent paracrine immunomodulatory factors with the capacity to deactivate the intestinal inflammatory response at multiple levels, and with potential therapeutic application in CD.

\section{ACKNOWLEDGEMENTS}

This work was supported by grants from the Spanish Ministry of Health (PI04/0674) and from Ramon Areces Foundation.

\section{Authors' affiliations}

E Gonzalez-Rey, A Fernandez-Martin, A Chorny, M Delgado, Institute of Parasitology and Biomedicine, CSIC, Granada, Spain

Conflict of interest: None declared.

\section{REFERENCES}

1 Fiocchi C. Inflammatory bowel disease: etiology and pathogenesis. Gastroenterology 1998;11:182-205.

2 Hanaver SB, Present DH. The state of the art in the management of inflammatory bowel disease. Rev Gastroenterol Disord 2003;3:81-92.

3 Elson CO, Sartor RB, Tennyson GS, et al. Experimental models of inflammatory bowel disease. Gastroenterology 2003;10:1344-67.

4 Strober W, Fuss IJ, Blumberg RS. The immunology of mucosal models of inflammation. Annu Rev Immunol 2002;20:495-49.

5 Vaughan J, Donaldson C, Bittencourt J, et al. Urocortin, a mammalian neuropeptide related to fish urotensin I and to corticotropin-releasing factor. Nature 1995;378:287-92.

6 Hinson JP, Kapas S, Smith DM. Adrenomedullin, a multifunctional regulatory peptide. Endocrin Rev 2000;21:138-67.

7 Oki Y, Sasano H. Localization and physiological roles of urocortin. Peptides 2004;25:1745-9.

8 Muramatsu Y, Fukushima K, lino K, et al. Urocortin and corticotropinreleasing factor receptor expression in the human colonic mucosa. Peptides 2000;21:1799-809.

9 Gravanis A, Margioris AN. The corticotropin-releasing factor (CRF) family of neuropeptides in inflammation: potential therapeutic applications. Curr Med Chem 2005; 12:1503-12.

10 Tsatsanis C, Androulidaki A, Dermitzaki E, et al. Urocortin 1 and Urocortin 2 induce macrophage apoptosis via CRFR2. FEBS Lett 2005;579:4259-64.

11 Saruta M, Takahashi K, Suzuki T, et al. Urocortin 1 in colonic mucosa in patients with ulcerative colitis. J Clin Endocrinol Metab 2004;89:5352-61.

12 Kageyama K, Bradbury MJ, Zhao L, et al. Urocortin messenger ribonucleic acid: tissue distribution in the rat and regulation in thymus by lipopolysaccharide and glucocorticoids. Endocrinology 1999; 140:5651-8.

13 Elsasser TH, Kahl S. Adrenomedullin has multiple roles in disease stress: development and remission of the inflammatory response. Microsc Res Techn 2002;57:120-9.

14 Ueda S, Nishio K, Minamino N, et al. Increased plasma levels of adrenomedullin in patients with systemic inflammatory response syndrome. Am J Respir Crit Care Med 1999; 160:132-6.

15 Kubo A, Minamino N, Isumi Y, et al. Production of adrenomedullin in macrophage cell line and peritoneal macrophage. J Biol Chem 1998;273:16730-8.

16 Poliak S, Mor F, Conlon P, et al. Stress and autoimmunity: the neuropeptides corticotropin-releasing factor and urocortin suppress encephalomyelitis via effects on both the hypothalamic-pituitary-adrenal axis and the immune system. J Immunol 1997;158:5751-6.

17 Agnello D, Bertini R, Sacco S, et al. Corticosteroid-independent inhibition of tumor necrosis factor production by the neuropeptide urocortin. Am J Physiol Endocrinol Metab 1998;275:E757-62.

18 Gonzalez-Rey E, Chorny A, Robledo G, et al. Urocortin and adrenomedullin prevent lethal endotoxemia by downregulating the inflammatory response. Am J Pathol 2006 (in press)

19 Turnbull AV, Vale W, Rivier C. Urocortin, a corticotropin-releasing factorrelated mammalian peptide, inhibits edema due to thermal injury in rats. Eur J Pharmacol 1996;303:213-6.

20 Chatzaki E, Charalampopoulos I, Leontidis C, et al. Urocortin in human gastric mucosa: relationship to inflammatory activity. J Clin Endocrinol Metab 2003;88:478-83.

21 Wong LY, Cheung BM, Li YY, et al. Adrenomedullin is both proinflammatory and antiinflammatory: its effects on gene expression and secretion of cytokines and macrophage migration inhibitory factor in NR8383 macrophage cell line. Endocrinology 2005; 146:1321-7.

22 Isumi Y, Kubo A, Katafuchi T, et al. Adrenomedullin suppresses interleukin1 beta-induced tumor necrosis factor-alpha production in Swiss 3 T3 cells. FEBS Lett 1999;463: 110-14

23 Neurath MF, Fuss I, Kelsall BL, et al. Antibodies to IL-12 abrogate established experimental colitis in mice. J Exp Med 1995;182:1281-90.

24 Fiorucci S, Mencarelli A, Palazzetti B, et al. Proteinase-activated receptor (PAR)-2 is an anti-inflammatory signal for colonic lamina propria lymphocytes in a mouse model of colitis. Proc Natl Acad Sci U S A 2001 ;98:13936-41.

25 Kihara N, De la Fuente SG, Fujino K, et al. Vanilloid receptor-1 containing primary sensory neurons mediate dextran sulphate sodium induced colitis in rats. Gut 2003;52:713-19.

26 Bradley PP, Priebat DA, Christensen RD et al. Cellular and extracellular myeloperoxidase in pyogenic inflammation. $J$ Invest Dermatol 1982;78:206-9. 
27 Uhlar CM, Whitehead AS. Serum amyloid A, the major vertebrate acutephase reactant. Eur J Biochem 1999;265:501-23.

28 Neurath MF, Finotto S, Glimcher LH. The role of Th1/Th2 polarization in mucosal immunity. Nat Med 2002;8:567-73.

29 Bacala R, Kono DH, Theofilopoulos AN. Interferons as pathogenic effectors in autoimmunity. Immunol Rev 2005;204:9-26.

30 McCormack G, Moriaty D, O'Donoghue DP, et al. Tissue cytokine and chemokine expression in inflammatory bowel disease. Inflamm Res 2001;50:491-5

31 Niederau C, Backmerhoff F, Schumacher B, et al. Inflammatory mediators and acute phase proteins in patients with Crohn's disease and ulcerative colitis. Hepatogastroenterology 1997;44:90-107.

32 Thompson C, Powrie F. Regulatory T cells. Curr Opin Pharmacol 2004:4:408-14.

33 Mills KH, McGuirk P. Antigen-specific regulatory T cells-their induction and role in infection. Semin Immunol 2004;16:107-17.

34 Singh B, Read S, Asseman C, et al. Control of intestinal inflammation by regulatory T cells. Immunol Rev 2001;182:190-200.

35 Groux H, O'Garra A, Bigler M, et al. A CD4+ T-cell subset inhibits antigenspecific T-cell responses and prevents colitis. Nature 1997;389:737-42.

36 Asseman C, Mauze S, Leach MW, et al. An essential role for interleukin 10 in the function of regulatory $T$ cells that inhibit intestinal inflammation. J Exp Med 1999;190:995-1004.

37 lellem A, Mariani M, Lang R, et al. Unique chemotactic response profile and specific expression of chemokine receptors CCR4 and CCR8 by CD4(+)CD25(+) regulatory T cells. J Exp Med 2001; 194:847-53.

38 Wang $L$, Wells $A D$, Dorf ME, et al. Recruitment of Foxp3+T regulatory cells mediating allograft tolerance depends on the CCR4 chemokine receptor. J Exp Med 2005;2011037-44.

39 Amstrong AM, Foulkes $R$, Jennings $G$, et al. Tumour necrosis factor inhibitors reduce the acute-phase response in hapten-induced colitis. Br J Surg $2001 ; 88: 235-40$.
40 Neurath MF, Fuss I, Pasparakis $M$, et al. Predominant pathogenic role of tumor necrosis factor in experimental colitis in mice. Eur J Immunol 1997; 27:1743-50

41 Fiorucci S, Mencarelli A, Palazzetti B, et al. Importance of innate immunity and collagen binding integrin $\alpha 1 \beta 1$ in TNBS-induced colitis. Immunity 2002; 17:769-80

42 Atreya R, Mudter J, Finotto S, et al. Blockade of interleukin 6 trans signaling suppresses T-cell resistance against apoptosis in chronic intestinal inflammation: evidence in Crohn's disease and experimental colitis in vivo. Nat Med 2000;6:183-8.

43 Correa SG, Riera CM, Spiess J, et al. Modulation of the inflammatory response by corticotropin-releasing factor. Eur J Pharmacol 1997;319:85-90.

44 Chrousos GP. The hypothalamic-pituitary-adrenal axis and immune-mediated inflammation. N Engl J Med 1995;332:1351-62.

45 Boehme SA, Gaur A, Crowe PD, et al. Immunosuppressive phenotype of corticotropin-releasing factor transgenic mice is reversed by adrenalectomy Cell Immunol 1997; 176:103-12.

46 Agelaki S, Tsatsanis C, Gravanis A, et al. Corticotropin-releasing hormone augments proinflammatory cytokine production from macrophages in vitro and in lipopolysaccharide-induced endotoxin shock in mice. Infect Immun 2002;70:6068-74.

47 Venihaki M, Dikkes $P$, Carrigan A, et al. Corticotropin-releasing hormone regulates IL- 6 expression during inflammation. J Clin Invest 2001; 108:1159-66.

48 La Fleur SE, Wick EC, Idumalla PS, et al. Role of peripheral corticotropinreleasing factor and urocortin II in intestinal inflammation and motility in terminal ileum. Proc Natl Acad Sci U S A 2005;102:7647-52.

49 Torpy DJ, Webster EL, Zachman EK, et al. Urocortin and inflammation: confounding effects of hypotension on measures of inflammation. Neuroimmunomodulation 1999;6:182-6.

\section{EDITOR'S QUIZ: GI SNAPSHOT}

\section{An unsuspected cause of chronic colitis}

\section{Clinical presentation}

A 38 year old man presented to our clinic with a four year history of histologically confirmed Crohn's colitis refractory to treatment and oligoarticular pain and swelling, especially of the knees, interpreted as Crohn's arthritis. Colitis had worsened and diarrhoea became frankly bloody after two years, despite various treatment combinations of topical and systemic mesalazine, prednisolone, sulfasalazine, and azathioprine.

In the patient's notes there was no history of travel abroad. The patient recalled that when symptoms started he had been working on cleaning water pipes of buildings.

At the time of presentation, inflammatory laboratory markers were moderately increased and white blood cell count revealed neutrophilic leucocytosis. Colonoscopy showed a pancolitis with ulcerations (size $2-3 \mathrm{~cm}$ ) gradually increasing in number from the caecum towards the rectum (fig 1). The ileum was not involved. Histology confirmed superficial colonic ulcerations with inflammatory hyperplasia of the colonic mucosa and hyperplastic colon polyps. Granulomata, fistula, or dysplastic cells were not found.

\section{Question}

What is the diagnosis?

See page 841 for answer

This case is submitted by:

J Richter

T Heintges

Clinics for Gastroenterology, Hepatology, and Infectious Diseases, University Clinics Düsseldorf, Heinrich-Heine-University, Düsseldorf, Germany

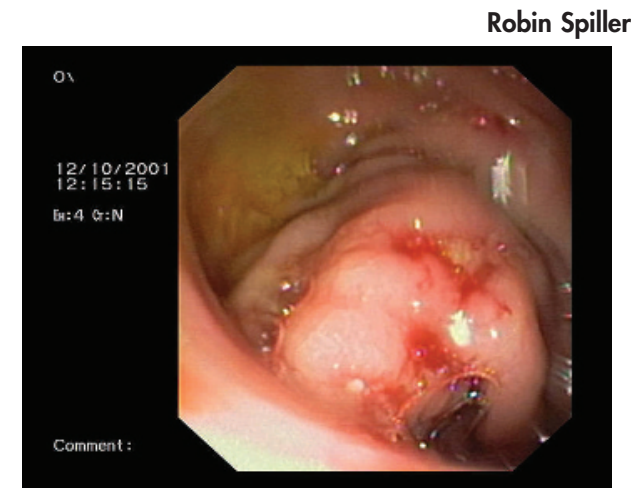

Figure 1 Endoscopic aspect of colitis.

Liebfrauenstr 10, D-47798 Krefeld, Germany

E Tannich

Bernhard-Nocht-Institute for Tropical Diseases, Hamburg, Germany

D Häussinger

Clinics for Gastroenterology, Hepatology, and Infectious Diseases, University Clinics Düsseldorf, Heinrich-Heine-University, Düsseldorf, Germany

Correspondence to: Dr J Richter, Tropenmedizinische Ambulanz, Klinik für Gastroenterologie, Hepatologie und Infektiologie, Universitätsklinikum Düsseldorf, Heinrich-Heine-Universität Düsseldorf, Moorenstraße 5, 40225 Düsseldorf, Germany; Joachim.Richter@med.uni-duesseldorf.de doi: 10.1136/gut.2005.077487 